Copyright by the Acoustical Society of America. Wang, B. T., Fuller, C. R., \& Dimitriadis, E. K. (1991). Active control of noise transmission through rectangular

plates using multiple piezoelectric or point force actuators. Journal of the Acoustical Society of America, 90(5), 2820-2830. doi: 10.1121/1.401879

\title{
Active control of noise transmission through rectangular plates using multiple piezoelectric or point force actuators
}

\author{
Bor-Tsuen Wang \\ Department of Mechanical Engineering, National Pingtung Polytechnic Institute, Pingtung, Taiwan 91207, \\ Republic of China \\ Chris R. Fuller and Emilios K. Dimitriadis \\ Department of Mechanical Engineering, Virginia Polytechnic Institute and State University, Blacksburg, \\ Virginia 24061
}

(Received 18 July 1990; revised 28 February 1991; accepted 23 May 1991)

This paper analytically demonstrates the use of multiple piezoelectric actuators bonded to the surface and point force actuators applied directly to a plate to reduce sound transmission through the plate. A harmonic plane wave incident on a simply supported, thin rectangular plate mounted in an infinite baffle was considered as the primary source. Both multiple piezoelectric and point force actuators are separately used as secondary (control) sources to attenuate the sound transmission through the plate. An optimal process was applied to obtain the input voltages of the piezoelectric actuators and the magnitude of the point forces, so that the radiated acoustic power can be minimized. Results show that a reduction of sound transmission through the plate is successfully achieved, if the proper size, number, and position of the piezoelectric or point force actuators are selected. Additionally, a comparison showed that point force actuators provide more effective control of the sound transmission than piezoelectric actuators; however, piezoelectric patches have more practical implementation than point force shakers, because of their low cost and light weight.

PACS numbers: 43.20.Rz, 43.40.Dx, 43.40.Vn, 43.88.Fx

\section{NOMENCLATURE}

C

$C_{0}$

$c$

$d_{31}$

E

$E_{a}$

F

$h$

$\bar{I}_{m}, \bar{I}_{n}$

$I_{m n}$

$K$

$L_{x}, L_{y}$

$N_{s}$

$N_{c}$

$N_{f}$

$P_{i}$

$P_{m n}$

$p_{i}$

$p_{t}$

$p_{n}$

$Q_{m n}$

$\bar{q}$

$=I_{m} I_{n}$ sound-pressure distribution function for piezoelectric actuators

sound-pressure distribution function for inci-

dent plane wave

sound-pressure distribution function for point

force actuators

material constant

sound speed in air

dielectric constant of piezoceramic patch

Young's modulus of plate

Young's modulus of piezoceramic patch

amplitude of point force

plate thickness

functions derived from Rayleigh's integral

functions of modal force for incident plane wave

function derived from Rayleigh's integral

plate dimensions

number of primary sources

number of piezoelectric actuators

number of point force actuators

amplitude of the incident pressure

modal force

incident plane-wave pressure

total sound pressure

sound pressure of primary source

modal function

amplitude vector of primary wave
$(R, \theta, \phi)$ polar coordinates of a point in the far-field

TL transmission loss

$t \quad$ time constant

$t_{a} \quad$ thickness of piezoelectric patch

$V \quad$ applied voltage to piezoelectric patch

$w \quad$ plate displacement

$W_{m n} \quad$ modal amplitude

$x, y \quad$ plate coordinates

$x_{f}, y_{f} \quad$ location of point force

$x_{1}, x_{2}, y_{1}, y_{2}$ location of piezoceramic patch

$\alpha_{m}, \beta_{n} \quad$ plate modal numbers

$\theta_{i}, \phi_{i} \quad$ incident angles of plane wave

$\Lambda \quad=d_{31} V / t_{a}$, free strain of piezoelectric patch

$v \quad$ Poisson ratio of plate

$v_{a} \quad$ Poisson ratio of piezoelectric patch

$\Pi_{i} \quad$ incident acoustic power

$\Pi_{t} \quad$ transmitted acoustic power

$k \quad \omega / c$, acoustic wave number

$\rho \quad$ mass density of air

$\rho_{p} \quad$ mass density of plate

$\Phi$ cost function

$\omega \quad=2 \pi f$, excitation frequency

$\omega_{m n} \quad$ plate natural frequency

\section{Superscripts}

$p_{i} \quad$ incident plane wave

$f \quad$ point force actuator

$c \quad$ piezoelectric actuator 


\section{INTRODUCTION}

Active control of sound transmission and radiation from vibrating structures has, in recent years, become a very interesting research area. The traditional method to attenuate the radiated sound is to use acoustic monopoles as active sources, as discussed in the paper by Deffayett and Nelson $^{1}$ and Fuller et al. ${ }^{2}$ Fuller has demonstrated both analytically ${ }^{3}$ and experimentally ${ }^{2}$ that applying external forces to the structure can successfully attenuate the radiated sound in a global sense using a low number of control transducers; however, electromagnetic shakers used as active inputs are generally heavy and cumbersome, requiring a reaction mount, and are thus not practical for use, especially in lightweight structures. It is in this control transducer area that piezoelectric actuators show much potential. These devices have been increasingly used in vibration control of large space structures because of their light weight and low cost. ${ }^{4} \mathrm{~A}$ significant advantage is that the piezoelectric elements may be either attached to or imbedded directly into the structure and thus overcome many of the point force handicaps.

It is in this context that piezoelectric actuators have been investigated for use as active control inputs (secondary sources) for reducing structure-borne sound radiation. Dimitriadis et al..$^{5}$ derived the equations for excitation of twodimensional structures, such as panels, with the use of piezoelectric actuators. They analytically demonstrated that the piezoelectric actuators can excite selected modes of vibration when used in a distributed sense (distributed here means a number of independent actuators or an actuator covering a relatively large area of the panel). Dimitriadis and Fuller ${ }^{6}$ also analytically showed that a single piezoelectric element can provide high attenuation of sound transmission through a clamped circular elastic plate. The potential of piezoelectric actuators for sound radiation control were also confirmed in some preliminary experiments by Fuller. ${ }^{7}$ More recently, Wang et al..$^{8,9}$ have developed an analytical model which considers active control of sound radiation from panels by multiple piezoelectric actuators as secondary sources. The primary source input consisted of a force applied to the structure. The results were very encouraging. It is again demonstrated that global control of sound radiation could be achieved with the piezoelectric elements employed as distributed actuators.

In this paper, harmonic plane sound waves incident on a simply supported thin rectangular plate are considered as primary sources. Either piezoelectric or point force actuators are employed as secondary (control) sources to reduce the sound transmission through the plate. An optimal control theory ${ }^{10}$ is adopted to optimize the input complex voltages to the piezoelectric or point force actuators so as to minimize the total radiated acoustic power from the plate. This has the effect of increasing the plate transmission loss. The performance of the piezoelectric and point force actuators is evaluated for various input frequencies, number, and location of control inputs. Finally, a comparison between the effectiveness of piezoelectric actuators versus point force actuators in terms of reduction in transmitted sound power is made. The investigation is thought to lay out the fundamental aspects of piezoelectric devices in terms of practical applications to active control of sound transmission. For example, sound attenuation of aircraft interior noise and machinery noise, as well as high-transmission-loss lightweight barriers, are typical applications of the technique.

\section{ANALYSIS}

\section{A. Incident wave, control inputs, and plate vibration}

A harmonic acoustic plane wave is considered incident on a simply supported, thin rectangular plate. Figure 1

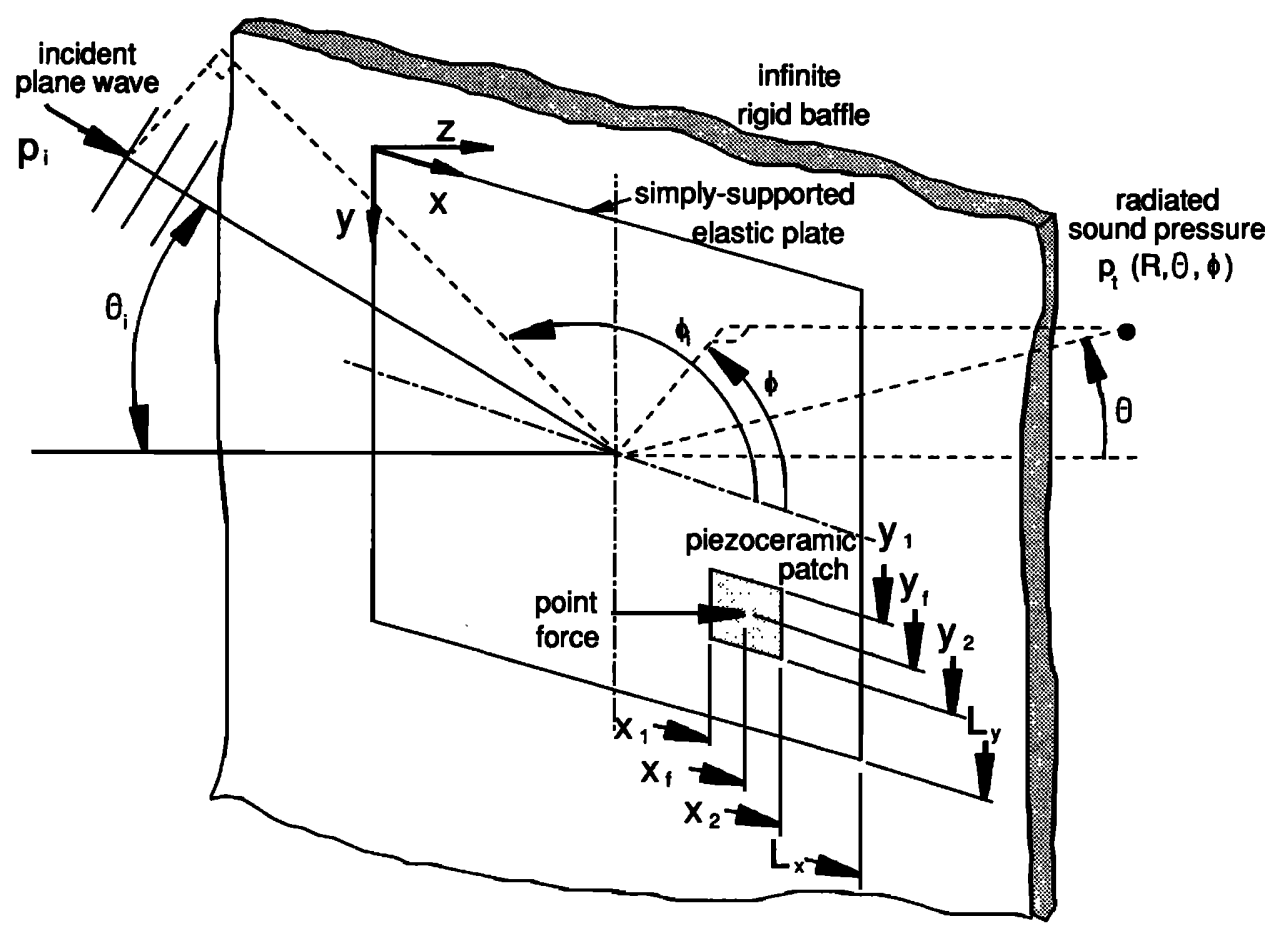

FIG. 1. Arrangement and coordinates of system. 
shows the arrangement and coordinates of the system. For the present analysis, the plate response is calculated for light fluid loading, and thus radiation loading effects on plate dynamics are ignored. The structural damping is also assumed to be negligible. The oblique incident plane wave is described by

$$
p_{i}(x, y, t)=P_{i} e^{i\left(\omega t-k_{x} \sin \theta_{i} \cos \phi_{i}-k_{y} \sin \theta_{i} \sin \phi_{i}\right)} .
$$

To calculate the radiated acoustic field, the plate vibration must be completely described. The plate displacement can be written as ${ }^{9}$

$$
w(x, y)=\sum_{m=1}^{\infty} \sum_{n=1}^{\infty} W_{m n} \sin \alpha_{m} x \sin \beta_{n} y,
$$

where

$$
\begin{aligned}
& \alpha_{m}=m \pi / L_{x}, \\
& \beta_{n}=n \pi / L_{y}, \\
& W_{m n}=\frac{P_{m n}}{\rho_{p} h\left(\omega_{m n}^{2}-\omega^{2}\right)} .
\end{aligned}
$$

Here, $P_{m n}$ is the modal force which depends on the exact description of the applied external load.

For an oblique incident plane wave, Roussos ${ }^{11}$ derived the modal force $P_{m n}^{p_{i}}$ (the superscript $p_{i}$ denotes the incident plane wave) as follows:

$$
P_{m n}^{p_{i}}=8 P_{i} \bar{I}_{m} \bar{I}_{n},
$$

where

$\bar{I}_{m}=-\frac{i}{2} \operatorname{sgn}\left(\sin \theta_{i} \cos \phi_{i}\right)$,

$$
\begin{gathered}
\text { if }(m \pi)^{2}=\left[\sin \theta_{i} \cos \phi_{i}\left(\omega L_{x} / c\right)\right]^{2}, \\
\bar{I}_{m}=\frac{m \pi\left\{1-(-1)^{m} \exp \left[-i \sin \theta_{i} \cos \phi_{i}\left(\omega L_{x} / c\right)\right]\right\}}{(m \pi)^{2}-\left[\sin \theta_{i} \cos \phi_{i}\left(\omega L_{x} / c\right)\right]^{2}} \\
\text { if }(m \pi)^{2} \neq\left[\sin \theta_{i} \cos \phi_{i}\left(\omega L_{x} / c\right)\right]^{2},
\end{gathered}
$$

and

$$
\begin{aligned}
\bar{I}_{n}= & -\frac{i}{2} \operatorname{sgn}\left(\sin \theta_{i} \sin \phi_{i}\right), \\
& \text { if }(n \pi)^{2}=\left[\sin \theta_{i} \sin \phi_{i}\left(\omega L_{y} / c\right)\right]^{2}, \\
\bar{I}_{n}= & \frac{n \pi\left\{1-(-1)^{n} \exp \left[-i \sin \theta_{i} \sin \phi_{i}\left(\omega L_{y} / c\right)\right]\right\}}{(n \pi)^{2}-\left[\sin \theta_{i} \sin \phi_{i}\left(\omega L_{y} / c\right)\right]^{2}}, \\
& \text { if }(n \pi)^{2} \neq\left[\sin \theta_{i} \sin \phi_{i}\left(\omega L_{y} / c\right)\right]^{2} .
\end{aligned}
$$

Two forms of control sources, piezpelectric and point force actuators, applied to the plate were considered. The corresponding expression of the modal force for piezoelectric excitation $P_{m n}^{c}$ was derived in Ref. 5 as

$$
\begin{aligned}
P_{m n}^{c}= & \frac{4 C_{0} \Lambda}{m n \pi^{2}}\left(\alpha_{m}^{2}+\beta_{n}^{2}\right)\left(\cos \alpha_{m} x_{1}-\cos \alpha_{m} x_{2}\right) \\
& \times\left(\cos \beta_{n} y_{1}-\cos \beta_{n} y_{2}\right),
\end{aligned}
$$

where $x_{1}, x_{2}, y_{1}$ and $y_{2}$ are the coordinates of the piezoelectric actuator, and the superscript $c$ signifies the piezoelectric actuator. The parameter $C_{0} \Lambda$ is defined in Ref. 5 for an actuator consisting of two identical piezoelectric patches bonded symmetrically on the two opposite plate faces and activated $180^{\circ}$ out-of-phase. $C_{0}$, a constant, is a function of material properties and dimensions given as follows:

$$
C_{0}=-E \frac{1+v_{a}}{1-v} \frac{P}{1+v-\left(1+v_{a}\right) P} \frac{2}{3}\left(\frac{h}{2}\right)^{2},
$$

where

$$
P=-\frac{E_{a}}{E} \frac{1-v^{2}}{1-v_{a}^{2}} \frac{3 t_{a}(h / 2)\left(h+t_{a}\right)}{2\left[(h / 2)^{3}+t_{a}^{3}\right]+3(h / 2) t_{a}^{2}}
$$

$\Lambda=d_{31} V / t_{a}$ is the strain induced by an unconstrained piezoelectric layer of thickness $t_{a}$, when a voltage $V$ is applied along its polarization direction, while $d_{31}$ is the dielectric strain constant of the piezoelectric.

The modal force for point force excitation $P_{m n}^{f}$ is given as follows:

$$
P_{m n}^{f}=\frac{4 F}{L_{x} L_{y}} \sin \alpha_{m} x_{f} \sin \beta_{n} y_{f},
$$

where $x_{f}$ and $y_{f}$ are the coordinates of the point force actuator, $F$ is the magnitude of the point force, and the superscript $f$ signifies the point force actuator.

\section{B. Sound radiation}

The sound radiation caused by the plate vibration is related to the acceleration distribution of the plate. Junger and Feit ${ }^{12}$ used the stationary-phase solution of Rayleigh's integral to derive a general expression for the sound pressure radiated from a vibrating panel. By superposition, their analysis can be extended to describe the sound radiation from a panel excited by various primary and control sources. Thus, for $N$, primary sources (harmonic incident plane waves), $N_{c}$ piezoelectric actuators, or $N_{f}$ point force actuators, the sound pressure radiated to a point $p(R, \theta, \phi)$ in the far field was derived to be, for primary sources,

$$
p_{n}(R, \theta, \phi)=K \sum_{j=1}^{N_{s}} \sum_{m=1}^{\infty} \sum_{n=1}^{\infty} W_{m n j}^{p_{i}} I_{m} I_{n},
$$

for piezoelectric control excitation,

$$
p_{c}(R, \theta, \phi)=K \sum_{j=1}^{N_{c}} \sum_{m=1}^{\infty} \sum_{n=1}^{\infty} W_{m n j}^{c} I_{m} I_{n},
$$

and for point force control excitation;

$$
p_{f}(R, \theta, \phi)=K \sum_{j=1}^{N_{f}} \sum_{m=1}^{\infty} \sum_{n=1}^{\infty} W_{m n j}^{f} I_{m} I_{n},
$$

where the constant $K$ and the quantities $I_{m}$ and $I_{n}$ can be found in Roussos ${ }^{11}$ as functions of $(R, \theta, \phi)$ :

$$
\begin{aligned}
K= & \frac{-\omega^{2} \rho L_{x} L_{y}}{2 \pi R} \\
& \times \exp \left[i w\left(t-\frac{r}{c}-\frac{\sin \theta}{2 c}\left(L_{x} \cos \phi+L_{y} \sin \phi\right)\right)\right],
\end{aligned}
$$

$$
\begin{aligned}
I_{m}= & -\frac{i}{2} \operatorname{sgn}(\sin \theta \cos \phi), \\
& \text { if }(m \pi)^{2}=\left[\sin \theta \cos \phi\left(\omega L_{x} / c\right)\right]^{2},
\end{aligned}
$$




$$
\begin{aligned}
I_{m}= & \frac{m \pi\left\{1-(-1)^{m} \exp \left[i \sin \theta \cos \phi\left(\omega L_{x} / c\right)\right]\right\}}{(m \pi)^{2}-\left[\sin \theta \cos \phi\left(\omega L_{x} / c\right)\right]^{2}} \\
& \text { if }(m \pi)^{2} \neq\left[\sin \theta \cos \phi\left(\omega L_{x} / c\right)\right]^{2}
\end{aligned}
$$

and

$$
\begin{aligned}
I_{n}= & -\frac{i}{2} \operatorname{sgn}(\sin \theta \sin \phi), \\
& \text { if }(n \pi)^{2}=\left[\sin \theta \sin \phi\left(\omega L_{y} / c\right)\right]^{2}, \\
I_{n}= & \frac{n \pi\left\{1-(-1)^{n} \exp \left[i \sin \theta \sin \phi\left(\omega L_{y} / c\right)\right]\right\}}{(n \pi)^{2}-\left[\sin \theta \sin \phi\left(\omega L_{y} / c\right)\right]^{2}},
\end{aligned}
$$

$$
\text { if }(n \pi)^{2} \neq\left[\sin \theta \sin \phi\left(\omega L_{y} / c\right)\right]^{2} \text {. }
$$

When the primary sources and piezoelectric actuators act simultaneously (i.e., control using piezoelectric actuators), the resulting sound-pressure field can be viewed as a superposition of the above given sound pressures for steady-state harmonic excitation. The total pressure can be conveniently written as

$$
p_{t}=p_{n}+p_{c}=\sum_{j=1}^{N_{s}}\left(P_{i}\right)_{j} B_{j}+\sum_{j=1}^{N_{c}}\left(C_{0} \Lambda\right)_{j} A_{j} .
$$

Similarly, when the incident plane wave and point force actuators act together (i.e., control using point force actuators), the total pressure can be written as

$$
p_{t}=p_{n}+p_{f}=\sum_{j=1}^{N_{s}}\left(P_{i}\right)_{j} B_{j}+\sum_{j=1}^{N_{f}} F_{j} C_{j},
$$

where $B_{j}, A_{j}$, and $C_{j}$ are the sound-pressure distribution functions for incident plane wave, piezoelectric, and point force actuators, respectively, given by

$$
\begin{aligned}
B_{j} & =K \sum_{m=1}^{\infty} \sum_{n=1}^{\infty} Q_{m n j}^{p_{i}} I_{m} I_{n}, \\
A_{j} & =K \sum_{m=1}^{\infty} \sum_{n=1}^{\infty} Q_{m n j}^{c} I_{m} I_{n},
\end{aligned}
$$

and

$$
C_{j}=K \sum_{m=1}^{\infty} \sum_{n=1}^{\infty} Q_{m n j}^{f} I_{m} I_{n},
$$

where

$$
\begin{aligned}
& Q_{m n j}^{p_{i}}=W_{m n j}^{P_{i}} /\left(P_{i}\right)_{g}, \\
& Q_{m n j}^{c}=W_{m n j}^{c} /\left(C_{0} \Lambda\right)_{j}, \\
& Q_{m n j}^{f}=W_{m n j}^{f} / F_{j} .
\end{aligned}
$$

\section{Optimal control}

Control of the primary field can be achieved by appropriately choosing the piezoelectric voltage parameters $C_{0} \Lambda$ or the point force magnitude $F$ to minimize the total radiated acoustic power into the far field. The cost function chosen to be minimized is the integral of the mean-squared sound pressure over a hemisphere of radius $R$ in the far field. ${ }^{3}$ This cost function, which is proportional to the radiated acoustic power, can be written as

$$
\Phi=\frac{1}{R^{2}} \int_{s}\left|p_{t}\right|^{2} d s=\int_{0}^{2 \pi} \int_{0}^{\pi / 2}\left|p_{t}\right|^{2} \sin \theta d \theta d \phi .
$$

When the expression for $p_{t}$ from Eqs. (19) or (20) is substi- tuted into Eq. (27), the cost function is obviously quadratic and possesses a unique minimum. A minimization procedure ${ }^{10}$ for the quadratic function was employed to calculate the optimal control parameters.

The total pressure of Eq. (19) (i.e., control using piezoelectric actuators) can be expressed in vector form

$$
p_{t}=\bar{B}^{T} \bar{q}+\bar{A}^{T} \bar{p},
$$

where

$$
\bar{B}=\left[\begin{array}{c}
B_{1} \\
B_{2} \\
\cdot \\
\cdot \\
\cdot \\
B_{N_{r}}
\end{array}\right]_{N_{s} \times 1},
$$

$$
\bar{A}=\left[\begin{array}{c}
A_{1} \\
A_{2} \\
\cdot \\
\cdot \\
\cdot \\
A_{N_{c}}
\end{array}\right]_{N_{c} \times 1},
$$

$$
\bar{q}=\left[\begin{array}{c}
\left(P_{i}\right)_{1} \\
\left(P_{i}\right)_{2} \\
\cdot \\
\cdot \\
\cdot \\
\left(P_{i}\right)_{N_{s}}
\end{array}\right]_{N_{s} \times 1} \text {, }
$$

$$
\bar{p}=\left[\begin{array}{c}
\left(C_{0} \Lambda\right)_{1} \\
\left(C_{0} \Lambda\right)_{2} \\
\cdot \\
\cdot \\
\cdot \\
\left(C_{0} \Lambda\right)_{N_{c}}
\end{array}\right]_{N_{c} \times 1}
$$

Then,

$$
\begin{aligned}
\left|p_{t}\right|^{2}= & \bar{p}^{T}\left[\bar{A} \bar{A}^{*^{T}}\right] \bar{p}^{*}+2 \operatorname{Re}\left(\bar{q}^{T}\left[\bar{B} \bar{A}^{*^{*}}\right] \bar{p}^{*}\right) \\
& +\bar{q}^{T}\left[\bar{B} \bar{B}^{{ }^{*} T}\right] \bar{q}^{*},
\end{aligned}
$$

where * denotes complex conjugate, and $T$ denotes transpose of matrix; hence the cost function can be written in matrix form as

$$
\begin{aligned}
\Phi= & \bar{p}^{T}[\widetilde{A}] \bar{p}^{*}+2 \operatorname{Re}\left(\bar{q}^{T}[\widetilde{B} A] \bar{p}^{*}\right) \\
& +\bar{q}^{T}[\widetilde{B}] \bar{q}^{*},
\end{aligned}
$$

where

$$
\begin{aligned}
& {[\widetilde{A}]_{N_{c} \times N_{c}}=\int_{0}^{2 \pi} \int_{0}^{\pi / 2}\left[\bar{A} \bar{A}^{* T}\right] \sin \theta d \theta d \phi,} \\
& {[\widetilde{B A}]_{N_{s} \times N_{c}}=\int_{0}^{2 \pi} \int_{0}^{\pi / 2}\left[\bar{B} \bar{A}^{* T}\right] \sin \theta d \theta d \phi,} \\
& {[\widetilde{B}]_{N_{s} \times N_{s}}=\int_{0}^{2 \pi} \int_{0}^{\pi / 2}\left[\bar{B} \bar{B}^{* T}\right] \sin \theta d \theta d \phi .}
\end{aligned}
$$

Since 


$$
\begin{gathered}
{\left[\bar{A} \bar{A}^{* T}\right]_{N_{c} \times N_{c}}=\left[\begin{array}{c}
A_{1} \\
A_{2} \\
\cdot \\
\cdot \\
\cdot \\
A_{N_{c}}
\end{array}\right]_{N_{c} \times 1} \times\left[A_{1}^{*} A_{2}^{*} \cdots A_{N_{c}}^{*}\right]_{1 \times N_{c}}} \\
=\left[\begin{array}{cccc}
A_{1} A_{1}^{*} & A_{1} A_{2}^{*} & A_{1} A_{N_{c}}^{*} \\
A_{2} A_{1}^{*} & A_{2} A_{2}^{*} & A_{2} A_{N_{c}}^{*} \\
\vdots & \vdots & \cdots & \vdots \\
A_{N_{c}} A_{1}^{*} & A_{N_{c}} A_{2}^{*} & A_{N_{c}}^{*} A_{N_{c}}^{*}
\end{array}\right]_{N_{c} \times N_{c}}
\end{gathered}
$$

a typical element of $\left[\bar{A}^{*}{ }^{* T}\right]$ is

$$
A_{r} A_{s}^{*}=K_{r} K_{s}^{*} \sum_{k=1}^{\infty} \sum_{l=1}^{\infty} \sum_{m=1}^{\infty} \sum_{n=1}^{\infty} Q_{k l r}^{c} Q_{m n s}^{c^{*}} I_{k l r}^{c} I_{m n s}^{c^{*}},
$$

and then, for a typical element of $[\tilde{A}]_{N_{c} \times N_{c}}$,

$\widetilde{A}_{r s}=\int_{0}^{2 \pi} \int_{0}^{\pi / 2} K_{r} K_{s}^{*} \sum_{k=1}^{\infty} \sum_{l=1}^{\infty} \sum_{m=1}^{\infty} \sum_{n=1}^{\infty} Q_{k l r}^{c} Q_{m n s}^{c^{*}} I_{k l r}^{c} I_{m n s}^{c^{*}}$

$\times \sin \theta d \theta d \phi$.

Similarly, a typical element of $[\tilde{B A}]_{N_{s} \times N_{c}}$ is as follows:

$$
\begin{aligned}
\tilde{B A_{r s}}= & \int_{0}^{2 \pi} \int_{0}^{\pi / 2} K_{r} K_{s}^{*} \sum_{k=1}^{\infty} \sum_{i=1}^{\infty} \sum_{m=1}^{\infty} \sum_{n=1}^{\infty} Q_{k l r}^{P_{i}} Q_{m n s}^{c^{*}} \\
& \times I_{k l r}^{P_{i}} I_{m n s}^{I^{*}} \sin \theta d \theta d \phi,
\end{aligned}
$$

and a typical element of $[\widetilde{B}]_{N_{s} \times N_{s}}$

$$
\begin{aligned}
\widetilde{B}_{r s}= & \int_{0}^{2 \pi} \int_{0}^{\pi / 2} K_{r} K_{s}^{*} \sum_{k=1}^{\infty} \sum_{i=1}^{\infty} \sum_{m=1}^{\infty} \sum_{n=1}^{\infty} Q_{k i r}^{P_{i}^{*}} Q_{m n s}^{P_{i l s}^{*}} I_{k i r}^{P_{i}} \\
& \times I_{m n \mathrm{r}}^{P_{i}^{*}} \sin \theta d \theta d \phi .
\end{aligned}
$$

Since $\left(\bar{q}^{T}[\widetilde{B}] \bar{q}^{*}\right)$ is a constant, the cost function can then be redefined as

$$
\widetilde{\Phi}=\Phi-\bar{q}^{T}[\widetilde{B}] \bar{q}^{*} .
$$

If we let

$$
\bar{F}^{T}=-\bar{q}^{T}[\tilde{B A}],
$$

then the optimal solution to minimize the cost function can be found as in Ref. 10:

$$
\bar{p}=[\tilde{A}]^{-1} \bar{F} \text {. }
$$

It is noted that $\bar{p}$ is the optimized vector which is defined in Eq. (32). Similarly, when point forces are used as control sources, one can easily find the corresponding optimal solution.

\section{Plate transmission loss}

For the incident acoustic plane wave at angle $\theta_{i}$ and $\phi_{i}$, the incident acoustic power is easily shown to be

$$
\Pi_{i}=P_{i}^{2} L_{x} L_{y} \cos \theta_{i} / 2 \rho c,
$$

where $\rho$ and $c$ are mass density of air and sound speed in air, and the radiated acoustic power from the plate (on the other side) is

$$
\Pi_{t}=\frac{\Phi R^{4}}{2 \rho c} .
$$

Then, transmission loss (TL) through the plate can be defined as follows:

$$
\mathrm{TL}=10 \log _{10}\left(\Pi_{i} / \Pi_{t}\right) .
$$

Transmission loss is an evaluation of the inverse of sound transmission efficiency; hence the larger the value of TL, the less sound power is transmitted through the panel with a corresponding improvement in reduction of global sound radiation.

\section{ANALYTICAL RESULTS}

Table I shows the physical properties of the rectangular plate which was used for illustrative results. Table II shows the natural frequencies of the simply supported plate for modes $(m, n)$. The optimal process is suitable for controlling multiple primary sources; however, only one harmonic incident plane wave with input parameters, $\theta_{i}=45^{\circ}, \phi_{i}=0^{\circ}$, and $P_{i}=10 \mathrm{~N} / \mathrm{m}^{2}$, was considered for the following results. It is assumed that the plate is radiating into air. Both the radiation directivity and plate displacement distribution were presented to demonstrate the control effectiveness of sound transmission by using piezoelectric or point force actuators. The following results consist of the plate displacement distribution plotted along the $y=L_{y} / 2$ horizontal plate midline. The results are normalized by the largest amplitude obtained in each case. Radiation directivity patterns are also presented across the $y=L_{y} / 2$ plane. For convenience, all $\theta$ angular positions to the left of the origin in the sound radiation directivity pattern plots correspond to $\phi=180^{\circ}$ far-field positions. Similarly, the right half of each plot corresponds to $\phi=0^{\circ}$. The radiated pressure is plotted in $\mathrm{dB}$ re: $20 \times 10^{-6} \mathrm{~Pa}$. In order to show clearly the radiation directivity pattern, the sound-pressure level for some control cases may appear as negative values, which implies that the absolute value is less than the reference value. The radiated power is expressed in $\mathrm{dB} r e: 10^{-12} \mathrm{~W}$. The integrals in the above equations were evaluated using the Simpson's onethird rule approach, and the infinite sums were truncated at $m=5$ and $n=5$; i.e., 25 modes were included in the analysis. This number of modes was found to provide sufficient convergence for the series in Eq. (2) and Eqs. (13)-(15). In particular, for the cases of low wave-number excitation considered here, the plate displacement and the radiated soundpressure amplitude have no more than $0.01 \%$ difference in comparison to those results which include 100 modes (i.e., $m=n=10$ ).

TABLE I. Plate specifications.

\begin{tabular}{lll}
\hline \hline$E=207 \times 10^{9} \mathrm{~N} / \mathrm{m}^{2}$ & $v=0.292$ & $L_{x}=0.38 \mathrm{~m}$ \\
$\rho_{p}=7870 \mathrm{~kg} / \mathrm{m}^{3}$ & $h=2 \mathrm{~mm}$ & $L_{y}=0.30 \mathrm{~m}$ \\
\hline
\end{tabular}


TABLE II. Natural frequencies of plate $(\mathrm{Hz})$

\begin{tabular}{crrrrr}
\hline \hline $\mathrm{m} \backslash \mathrm{n}$ & \multicolumn{1}{l}{1} & \multicolumn{1}{c}{2} & \multicolumn{1}{c}{3} & \multicolumn{1}{c}{4} & \multicolumn{1}{c}{5} \\
\hline 1 & 87.71 & 249.81 & 519.98 & 898.22 & 1384.53 \\
2 & 188.74 & 350.85 & 621.02 & 999.25 & 1485.56 \\
3 & 357.13 & 519.23 & 789.40 & 1167.64 & 1653.95 \\
4 & 592.88 & 754.98 & 1025.15 & 1403.39 & 1889.69 \\
5 & 895.98 & 1058.08 & 1328.25 & 1706.48 & 2192.79 \\
\hline \hline
\end{tabular}

\section{A. Effect of number of control sources}

\section{Piezoelectric actuators}

Figure 2 shows the radiation directivity for the primary input consisting of an incident plate wave with circular frequency $85 \mathrm{~Hz}$ near the $(1,1)$ mode resonance, controlled by one, two, and three piezoelectric actuators, respectively. The locations and size of the piezoelectric actuators are sketched to scale on the top of Fig. 2. The primary sound radiation directivity denoted by the solid line reveals a uniform pattern at all angles, i.e., the characteristic shape of the $(1,1)$ mode. For one piezoelectric actuator located in the middle of the plate, the $(1,1)$ mode is well controlled. The remaining significant residual pattern is close to that associated with the $(2,1)$ plate mode, as shown in Fig. 2.

For two independently controlled piezoelectric actuators, as illustrated on the top of Fig. 2, the actuators can control not only the $(1,1)$ mode, but also the $(2,1)$ mode, so that the $(3,1)$ mode becomes the dominant mode. When three independently controlled piezoelectric actuators are applied as shown in Fig. 2, the actuators can simultaneously control several modes such as modes $(1,1),(2,1)$, and $(3,1)$; thereafter, the remaining dominant radiating mode is a combination of modes $(1,1)$ and $(3,1)$. The total reduction of radiated acoustic power shown in Table III is $76.2 \mathrm{~dB}$ for one piezoelectric actuator, $79.4 \mathrm{~dB}$ for two, and $96.8 \mathrm{~dB}$ for three. It may be concluded that the increasing numbers of actuators lead to an increase in reduction of radiated acous-
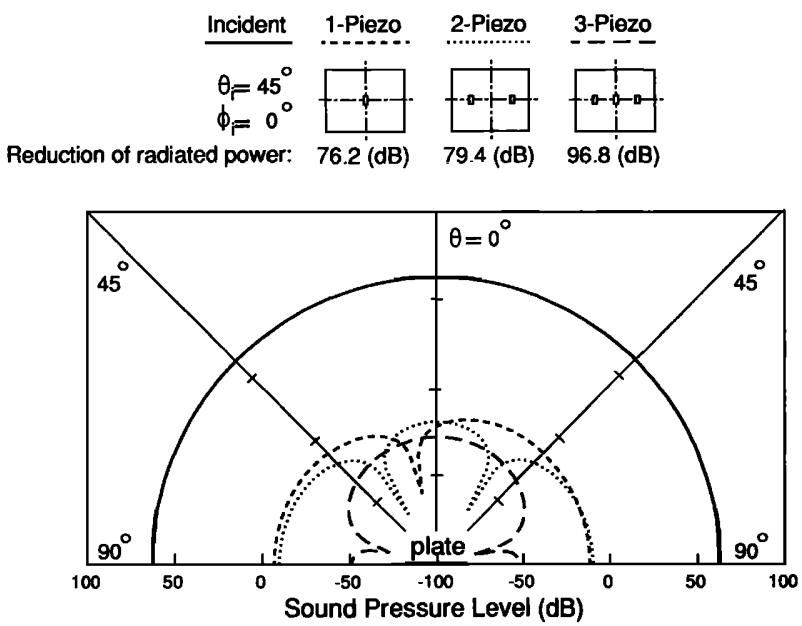

FIG. 2. Radiation directivity for a panel excited by a single-frequency ( 85 $\mathrm{Hz}$ ) incident plane wave and controlled by one, two, or three piezoceramic patches.
TABLE III. Total reduction of radiated acoustic power $(\mathrm{dB})$.

\begin{tabular}{ccccccc}
\hline \hline $\begin{array}{c}\text { Number } \\
\text { of } \\
\text { actuators }\end{array}$ & $\begin{array}{c}c=85 \mathrm{~Hz} \\
\text { Piezo- } \\
\text { ceramic } \\
\text { patch }\end{array}$ & $\begin{array}{c}\text { Point } \\
\text { force }\end{array}$ & $\begin{array}{c}f=140 \mathrm{~Hz} \\
\text { Piezo- } \\
\text { ceramic } \\
\text { patch }\end{array}$ & $\begin{array}{c}\text { Point } \\
\text { force }\end{array}$ & $\begin{array}{c}f=190 \mathrm{~Hz} \\
\text { Piezo- } \\
\text { ceramic } \\
\text { patch }\end{array}$ & $\begin{array}{c}\text { Point } \\
\text { force }\end{array}$ \\
\hline 1 & 76.2 & 76.3 & 34.5 & 34.6 & 0 & 0 \\
2 & 79.4 & 92.8 & 39.5 & 54.5 & 28.8 & 48.7 \\
3 & 96.8 & 108.0 & 60.1 & 70.8 & 56.7 & 66.7 \\
\hline \hline
\end{tabular}

tic power. For all cases, however, significant reduction in the radiated sound-pressure levels is demonstrated. For this case, the practical limit of attenuation (because of experimental limitation due to background noise, calibration accuracy, controller accuracy, etc.) is seen to be achieved with just one actuator. The companion experiments ${ }^{13}$ generally compare well with theory and are not shown here for brevity.

Figure 3 presents the plate displacement distribution corresponding to the cases of Fig. 2. These distributions, partially decomposed into modal amplitudes of varying $m$ with $n=1$, are also given in Table IV. The modal amplitude of the $(m, n)$ mode expressed in $\mathrm{dB}$ is normalized by that of the $(1,1)$ mode due to the incident plane wave alone. As expected, the $(1,1)$ mode dominates the plate vibration, because the incident plane wave is harmonically excited near the $(1,1)$ mode resonance frequency. When one piezoelectric is employed, the $(1,1)$ mode is well controlled; however, the significant energy is spilled into the $(3,1)$ mode, and the amplitude of the $(3,1)$ mode is raised. The residual plate displacement distribution thus takes on the shape of the $(3,1)$ mode. Note, however, that the $(2,1)$ amplitude is unchanged because of the central location of the single actuator. This result accounts for the sound radiation pattern observed in Fig. 2. When two actuators are employed, the $(2,1)$ amplitude is now reduced, but further control spillover occurs into the $(3,1)$. Nevertheless, the total sound power radiated falls. Two control characteristics due to applying se-
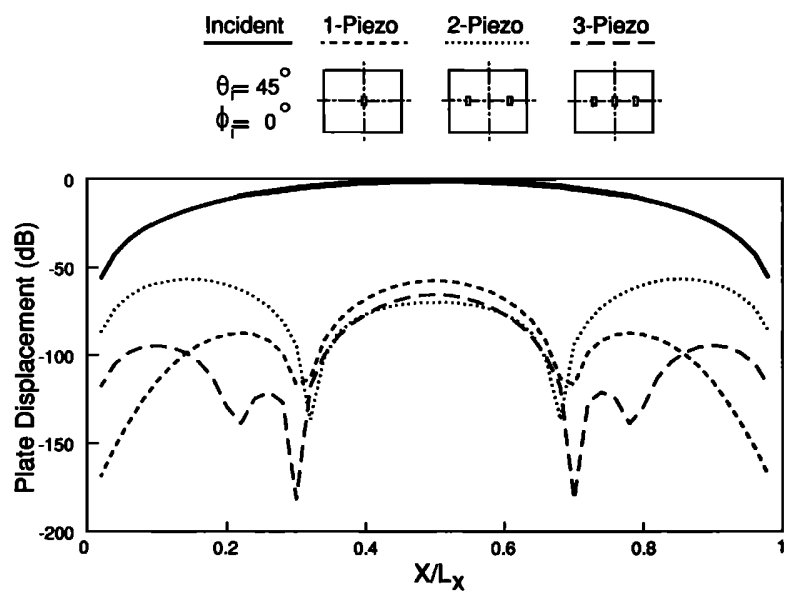

FIG. 3. Plate displacement for a panel excited by a single-frequency ( 85 $\mathrm{Hz}$ ) incident plane wave and controlled by one, two, or three piezoceramic patches. 
TABLE IV. Modal amplitude of plate vibration $(\mathrm{dB}), f=85 \mathrm{~Hz}$.

\begin{tabular}{rrrcc}
\hline \hline$m$ & $\begin{array}{c}\text { Incident } \\
n=1\end{array}$ & $\begin{array}{c}\text { One piezo } \\
n=1\end{array}$ & $\begin{array}{c}\text { Two piezo } \\
n=1\end{array}$ & $\begin{array}{c}\text { Three piezo } \\
n=1\end{array}$ \\
\hline 1 & 0.0 & -43.1 & -43.2 & -48.2 \\
2 & -55.3 & -55.3 & -89.7 & -89.7 \\
3 & -57.9 & -35.4 & -30.5 & -43.7 \\
4 & -83.0 & -82.9 & -68.9 & -68.9 \\
5 & -78.7 & -44.8 & -45.0 & -44.9 \\
\hline \hline
\end{tabular}

condary sources are observed here. First, the plate response is globally reduced, leading to attenuation of sound radiation, corresponding to what is termed "modal suppression."2 Second, the plate displacement appears as a highermode response with a low radiation efficiency, leading to less power radiated, corresponding to what is termed "modal restructuring."2 Finally, when three actuators are used, the $(1,1)$ and $(2,1)$ amplitudes remain attenuated, and control spillover to the $(3,1)$ is now observed in Table IV. This is also reflected in the residual displacement, as shown in Fig. 3. The displacement distribution appears to have a shape, due to the response, of many higher-order modes. This higher-mode plate response results in volumetric cancellation and thus explains the high-power reduction as observed in Fig. 2.

For the next results, the circular frequency of the primary input was increased to $190 \mathrm{~Hz}$, near the resonance of the $(2,1)$ mode. Figure 4 shows the resultant radiation directivity for one, two, and three piezoelectric actuators with the same size and locations as those in Fig. 2. As can be seen, the primary field appears to have the shape of a distorted $(2,1)$ mode. This is logical, since the excitation frequency is near the $(2,1)$ resonance point, and thus the $(2,1)$ mode dominates the response. This is supported by the results of Table $\mathrm{V}$ which are contributions of plate modes to the total radiated sound pressure at $R=10 \mathrm{~m}$. The $\mathrm{dB}$ reference is the maximum value of the uncontrolled transmitted pressure ampli-

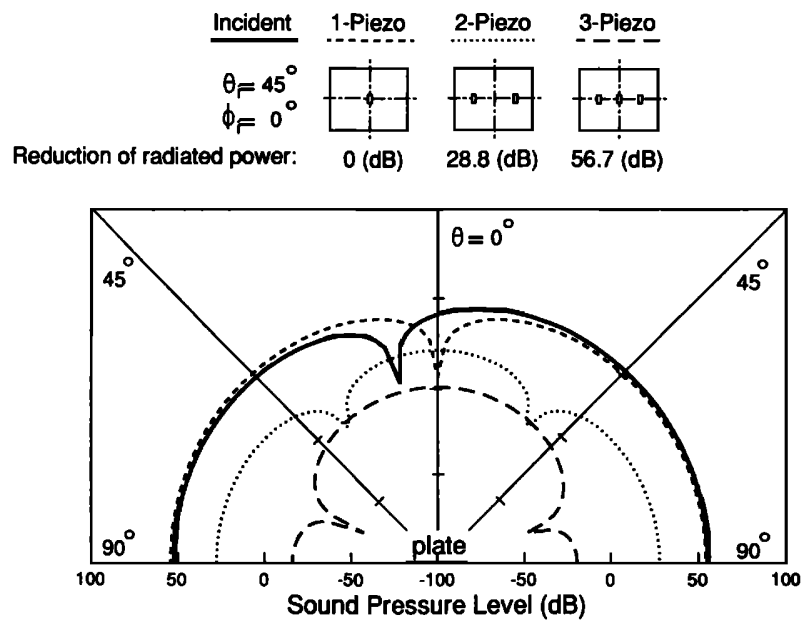

FIG. 4. Radiation directivity for a panel excited by a single-frequency ( 190 $\mathrm{Hz}$ ) incident plane wave and controlled by one, two, or three piezoceramic patches.
TABLE V. Modal amplitude of sound pressure (dB), $f=190 \mathrm{~Hz}$.

\begin{tabular}{rrrrr}
\hline \hline$m$ & $\begin{array}{c}\text { Incident } \\
n=1\end{array}$ & $\begin{array}{c}\text { One piezo } \\
n=1\end{array}$ & $\begin{array}{c}\text { Two piezo } \\
n=1\end{array}$ & $\begin{array}{c}\text { Three piezo } \\
n=1\end{array}$ \\
\hline 1 & -13.1 & -22.0 & -9.9 & -26.5 \\
2 & 0.0 & 0.0 & -68.6 & -68.6 \\
3 & -43.1 & -23.6 & -7.3 & -31.8 \\
4 & -68.9 & -68.9 & -54.5 & -54.5 \\
5 & -70.5 & -39.9 & -28.4 & -38.1 \\
\hline \hline
\end{tabular}

tude. This table reveals that the $(2,1)$ mode is indeed dominant, and the next most important mode is the $(1,1)$; both modes account for the distorted radiation directivity pattern due to the oblique incident plane wave $\theta_{i}=45^{\circ}$ and $\phi_{i}=0^{\circ}$.

When one piezoelectric actuator is used, Table $\mathrm{V}$ shows that the $(1,1)$ contribution is reduced. This is supported by Fig. 4, which now shows a symmetric radiation pattern similar to the $(2,1)$, thus confirming the removal of the $(1,1)$ contribution. However, there again has been significant spillover into the $(3,1)$ mode, which accounts for the mode at $\theta=0^{\circ}$ not being identically zero. The $(2,1)$ mode is not controllable because of the central location of the actuator.

The number of actuators were again increased to two, and now significant control of the $(2,1)$ contribution is observed both in Fig. 4 and Table V. This is, of course, due to the location of the two piezoelectric elements, which can now couple into the $(2,1)$ mode. Note that the residual field in this case has the characteristic shape of the $(3,1)$ mode. On increasing the number of actuators to three, control is now achievable over the $(1,1),(2,1)$, and $(3,1)$ contributions simultaneously, and large reductions are achieved.

Figure 5 gives the displacement distribution corresponding to the cases of Fig. 4. It is interesting to note that little change occurs in the displacement distribution when one control element is used, although significant change in the radiation field are observed. This result is due to the fact that the $(1,1)$ mode has a much higher radiation efficiency than the $(2,1)$; thus the displacement response of the $(1,1)$
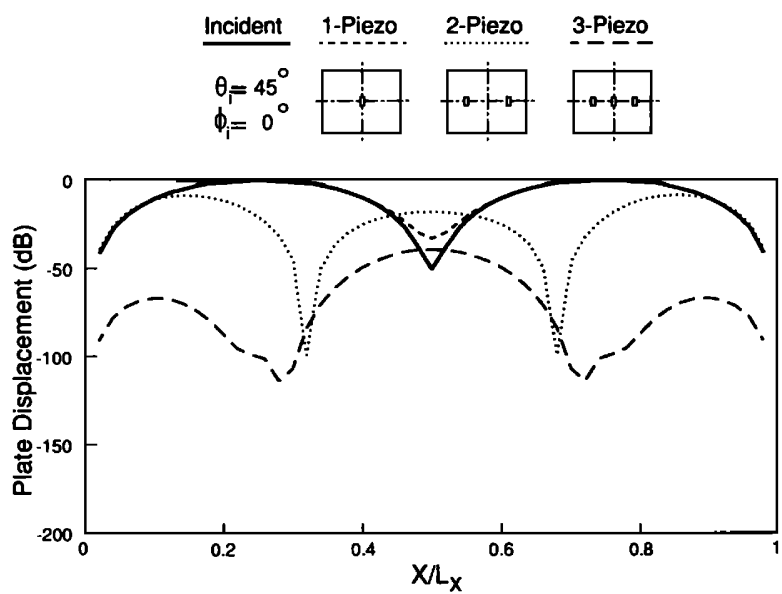

FIG. 5.Plate displacement for a panel excited by a single-frequency ( 190 $\mathrm{Hz}$ ) incident plane wave and controlled by one, two, or three piezoceramic patches. 
mode may be far lower than that of the $(2,1)$ mode. However, the $(1,1)$ mode can still contribute significantly to the radiated field. Hence small changes in plate response can lead to large changes in the radiated field. It is also apparent that the total plate response increases at $x / L_{x}=0.5$, and this is due to spillover into the $(3,1)$ mode. However, this is again not manifest in an increase in radiated levels due to the phenomenon of "modal restructuring."2 The characteristic of modal restructuring implies that when control is applied, the plate response is not globally reduced, but possibly even increased and changed to a higher-order response. This higher-order plate response generally has a smaller radiation efficiency: therefore, sound radiation from the plate is eventually attenuated, even though plate response has increased. Similar results can be observed for other actuator configurations in Fig. 5 and correspond well to what is seen in Fig. 4 and Table V.

\section{B. Comparison between piezoelectric and point force actuators}

It will be of great interest to compare the control performance between piezoelectric and point force actuators. Piezoelectric actuators have been recently introduced to active structural acoustic control, while point force actuators are customarily used. The radiation directivity patterns and displacement distributions for using point force actuators were found to be similar to those for using piezoelectric actuators in Figs. 2-5. Thus these results were not shown; instead, a comparison between piezoelectric and point force actuators in terms of control performance was made. In order to compare the control effectiveness of piezoelectric actuators with that of point force actuators, the point force was chosen to be located at the center of a piezoceramic patch. The effects of size and location of the piezoceramic patch, however, were not addressed in this paper. Wang et al. ${ }^{9}$ discussed these effects and demonstrated that the locations of actuators were best chosen where the plate has the largest out-of-plane response. Here, the radiation directivity, plate displacement distributions, and total reduction of radiated acoustic power were shown to evaluate the relative performance and piezoelectric and point force actuators.

\section{One actuator}

Figure 6 shows the radiation directivity for a frequency of $85 \mathrm{~Hz}$ near the $(1,1)$ mode resonance point. In this case, the control achieved by a single centrally located piezoelectric element and point force actuator were compared. Both the piezoelectric and point force actuators have nearly the same control effectiveness of sound radiation; however, the results of Fig. 7, which are the plate displacement distribution, indicates that the point force actuator gives better control in plate displacement, and its residual amplitude is less than that of the piezoelectric element. This can be interpreted as the point force actuator leading to less control spillover than the piezoelectric element, contrary to what was previously understood about distributed actuators. The reason for this behavior is not presently understood; however, it is under investigation. Some potential ideas will be discussed later.

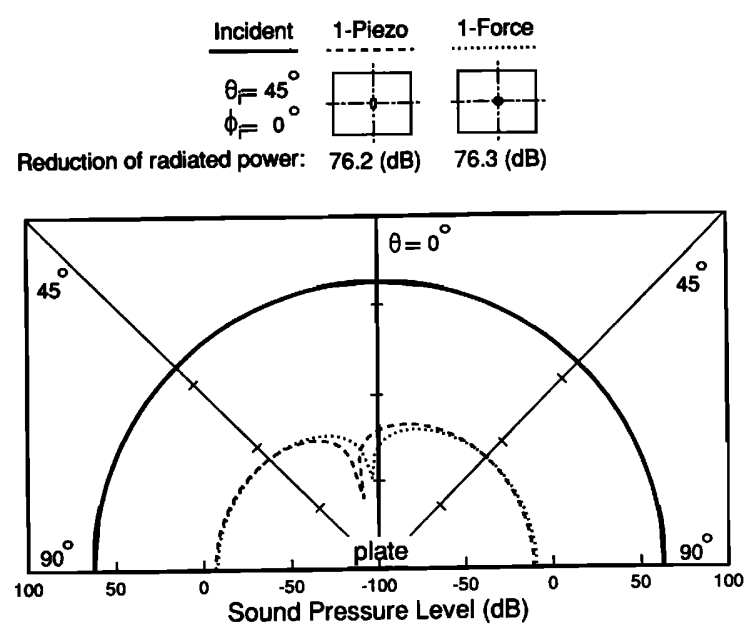

FIG. 6. Radiation directivity for a panel excited by a single-frequency ( 85 $\mathrm{Hz}$ ) incident plane wave and controlled by one point force or piezoceramic patch.

It is noted, from Figs. 6 and 7, that even though the "force" result is approximately $30 \mathrm{~dB}$ lower in plate response than "piezo" result, the residual radiation directivity are nearly the same level. The difference in radiation characteristic between the two results is what is termed "modal restructuring,"2 whereby plate response may be increased, but radiation falls. In this case, the residual vibration distribution for applying the point force actuator has a much higher radiation efficiency than applying the piezoelectric actuator.

\section{Two actuators}

In the next comparison, the circular frequency was increased to $190 \mathrm{~Hz}$ near the $(2,1)$ plate resonance frequency. In this case, as shown in Fig. 8, the $(2,1)$ mode dominates the radiation field with significant contribution from the $(1,1)$ mode, and the point force actuators clearly out perform the piezoelectric actuators in terms of reduction of radiated levels. This was also observed in Fig. 9, which shows plots of the corresponding displacement distributions. It is

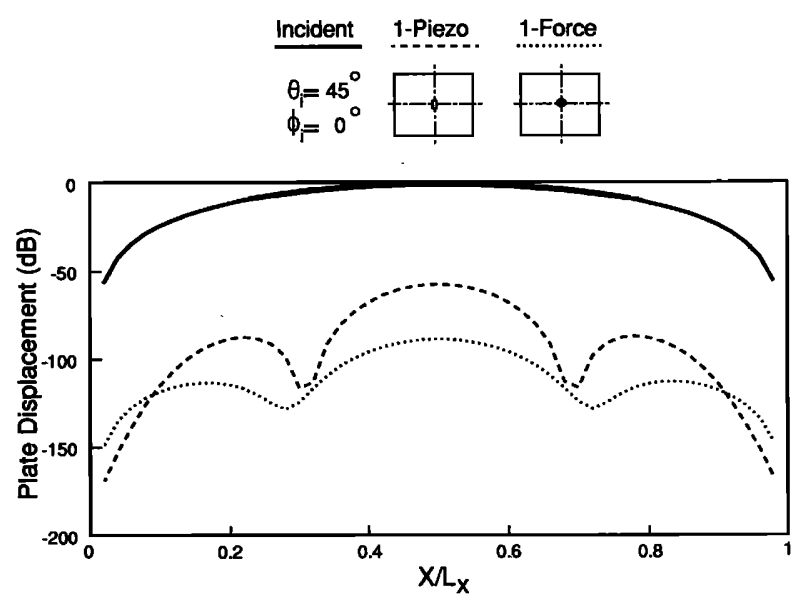

FIG. 7. Plate displacement for a panel excited by a single-frequency ( 85 $\mathrm{Hz}$ ) incident plane wave and controlled by one point force or piezoceramic patch. 


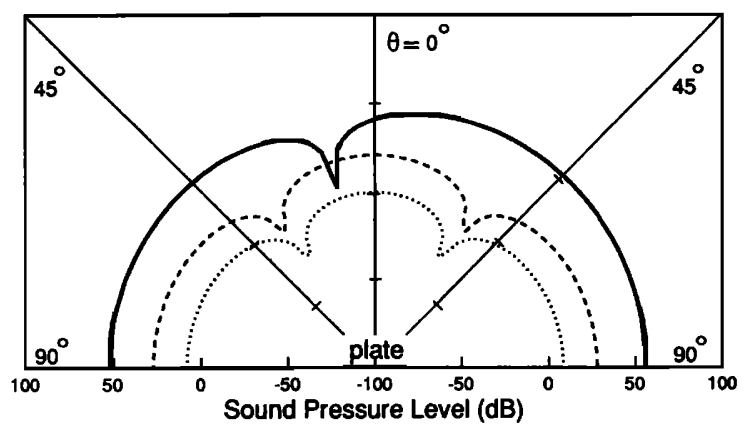

FIG. 8. Radiation directivity for a panel excited by a single-frequency (190 $\mathrm{Hz}$ ) incident plane wave and controlled by two point forces or piezoceramic patches.

apparent that the use of point force actuators has again led to significantly less spillover into the residual $(3,1)$ mode than the use of piezoelectric actuators.

\section{Three actuators}

For a stringent comparison test, the circular frequency is now reduced to $140 \mathrm{~Hz}$, which is off-resonance between the $(1,1)$ and $(2,1)$ mode resonance frequencies. Being offresonance, it is expected that more modes can contribute to the plate response and radiated field, thus exacerbating control spillover effects. Figures 10 and 11 give the radiation directivities and displacement distribution of this frequency. Again, it is apparent that the point control forces lead to less spillover and improved control performance, in terms of reduction of radiated sound level, than piezoelectric excitations.

Finally, Table III summarizes the total reduction of radiated acoustic power for the three different excitation frequencies and control by either one, two, or three piezoelec-
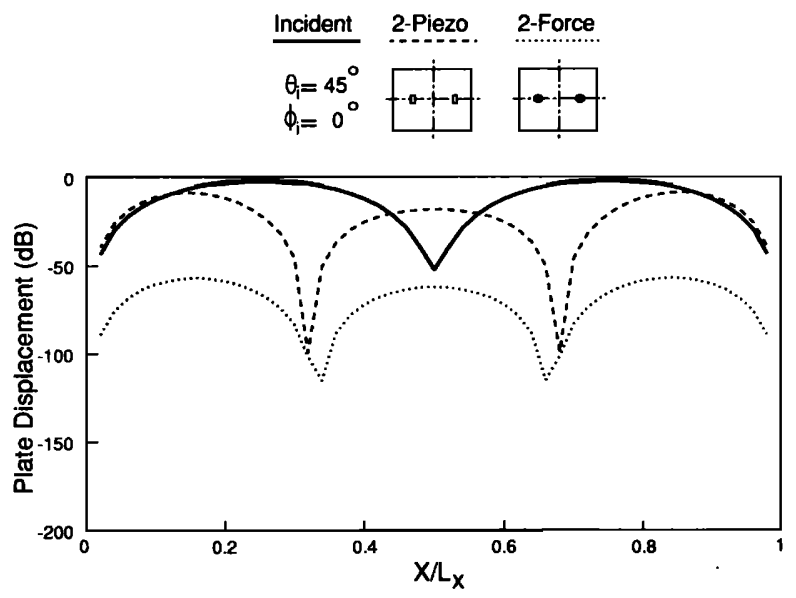

FIG. 9. Plate displacement for a panel excited by a single-frequency ( 190 $\mathrm{Hz}$ ) incident plane wave and controlled by two point forces or piezoceramic patches.
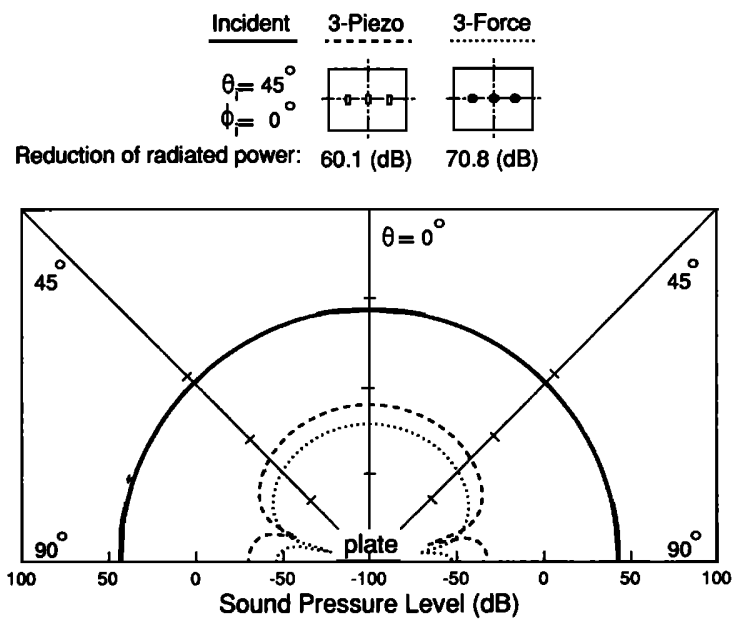

FIG. 10. Radiation directivity for a panel excited by a single-frequency ( 140 $\mathrm{Hz}$ ) incident plane wave and controlled by three point forces or piezoceramic patches.

tric patches or point forces. These results confirm that point forces indeed give better performance on a global basis than the piezoelectric elements. As stated previously, this result is somewhat contrary to what was expected. Distributed control (i.e., in this case, piezoelectric elements) is expected to give improved performance because of the fact that the distributed element can couple into less modes leading to less spillover. However, on-going work at VPI\&SU stimulated by this result tends to point to this distributed nature being a disadvantage rather than an advantage. The piezoelectric actuator generates only line moments along the edges of the actuator instead of the form of distributed inputs over the area of the piezoceramic patch. The present work has suggested that sound reduction occurs by the plate system assuming new eigenvalues and eigenfunctions (mode shapes) under feedforward control similar to the work on feedforward control of vibrations in beams discussed in Ref. 14 . Highest reduction in sound levels is achieved by creating new modes with the lowest total response and/or radiation efficiency. In this case, a point force is an ideal actuator, as it 
is equally coupled to all uncontrolled modes in the wavenumber domain, while the piezoelectric element has reduced coupling and results in a reduced range of achievable modal modification (i.e., the degree of modal restructuring is limited). However, this topic is out of the context of this paper and will be the subject of another publication, ${ }^{15}$ which presents the near-field response as well as wave-number domain analysis. It is also interesting to note that the original concepts concerning the improved performance with distributed control were made from studies which considered an infinite number of point forces distributed over a beam. ${ }^{16}$ Although piezoceramics are in a sense distributed, they exert a constant control input over finite regions of the structure, which is significantly different from the configuration of Meirovitch and Norris, ${ }^{16}$ and this characteristic is believed to lead to the different conclusion observed in this work.

\section{Transmission loss}

Figure 12 shows the transmission loss over the frequency range of $10-1000 \mathrm{~Hz}$ for the incident plane wave as the primary input and involving four separated cases of comparative control. For the controlled cases, the heavy lines correspond to piezoelectric actuators, while the light lines correspond to point force control.

For the incident plane wave, the transmission loss can be seen to dip at the resonance frequencies of the plate. It can be seen that the use of two forms of actuators leads to increased transmission loss over the frequency range except at a number of frequencies corresponding to asymmetric modes in modal number $n$. These modes have nodal lines at the actuator locations and are thus uncontrollable.

Figure 12 also exhibits an interesting behavior. As the number of actuators is increased, the transmission loss is seen to increase. The dips which indicate the resonant frequencies of the controlled plate system have been shifted to higher frequencies. These shifted dips can be possibly visualized as the new eigenproperties of the controlled plate system as studied by Burdisso and Fuller ${ }^{14}$ for feedforward control of a one-dimensional beam. One would expect that the transmission loss would dip at the new eigenvalues or reso-
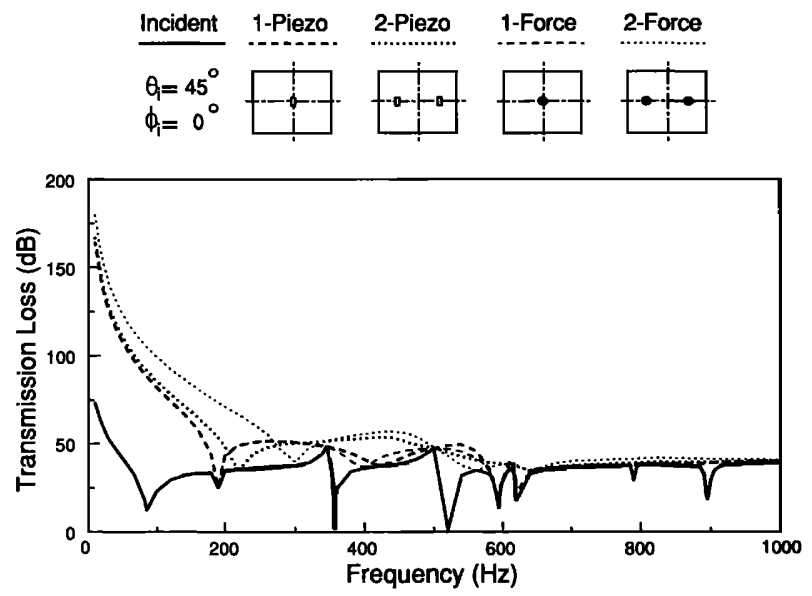

FIG. 12. Plate transmission loss versus frequency. nance frequencies of the closed-loop system. It is thought that the phenomenon investigated by Burdisso and Fuller ${ }^{14}$ is occurring here.

For the single actuator, the piezoelectric and point force actuators give about the same performance especially at lower frequencies. However, some differences are observed about the $(3,1)$ resonance for the reasons discussed above.

Figure 12 demonstrates that with two actuators used, the plate transmission loss is maintained at approximately $50 \mathrm{~dB}$ (greater than the limit of practical realization) from 0 to $650 \mathrm{~Hz}$ (i.e., $0<\kappa L_{x} L<4.52$ ). In this frequency range, nine different modes participate. Although it is difficult to generalize, the authors believe that two actuators will provide sufficient control in the low-frequency region defined by $\kappa L_{x} \leqslant \pi$. From Fig. 12, when one or two actuators are applied, plate transmission loss is adequately increased over a range of $0-650 \mathrm{~Hz}$, which includes nine modes. In other words, adequate control can be achieved by one or two actuators over a range including the first nine modes of the uncontrolled plate system.

\section{CONCLUSIONS}

The active control of a harmonic plane sound wave transmitting through a rectangular panel at an angle has been analytically studied. Both piezoelectric and point force actuators are considered, while the control cost function is derived from the far-field radiated acoustic power. The performance of the control system for an increasing number of control inputs is studied, and the attenuations obtained for point force and piezoelectric actuators are compared. The results show that both piezoelectric and point force actuators provide high reductions of sound transmitted through the plate if the proper size, number, and location of actuators are chosen. In general, as the number of actuators is increased, higher reductions are observed.

A very interesting result observed was that point force actuators were seen to perform slightly better than piezoelectric actuators. This result is contrary to present beliefs about distributed actuators and is presently under detailed investigations. However, piezoelectric actuators possess a number of advantages, such as lightweight, low cost, and compactness, which point force transducers cannot compete with. The study thus indicates that piezoelectric patch-type actuators show much potential for active control of sound and vibration.

\section{ACKNOWLEDGMENT}

The authors acknowledge the support by the Office of Naval Research under Grant ONR-N00014-88-K-0721.

${ }^{1}$ C. Deffayet and P. A. Nelson, "Active control of low frequency harmonic sound radiated by a finite panel,” J. Acoust. Soc. Am. 84, 2192-2199 (1988).

${ }^{2}$ C. R. Fuller, C. H. Hansen, and S. D. Snyder, "Active control of sound radiation from a vibrating rectangular panel by sound sources and vibration inputs: An experimental comparison," J. Sound Vib. 145(2), 195215 (1991).

${ }^{3}$ C. R. Fuller, "Active control of sound transmission radiation from elastic 
plates by vibration inputs. I. Analysis," J. Sound Vib. 136, 1-15 (1990). ${ }^{4}$ E. F. Crawley, and J. de Luis, "Use of piezoelectric actuators as elements of intelligent structures," AIAA J. 25, 1373-1385 (1987).

${ }^{5}$ E. K. Dimitriadis, C. R. Fuller, and C. A. Rogers, "Piezoelectric actuators for distributed noise and vibration excitation of thin plates," J. Vib. Acoust. 113, 100-107 (1991).

${ }^{6} \mathrm{E}$. K. Dimitriadis and C. R. Fuller, "Investigation on active control of sound transmission through elastic plates using piezoelectric actuators," AIAA paper 89-1062 (1989).

${ }^{7}$ C. R. Fuller, "Analysis of active control of sound radiation from elastic plates by force inputs," in Proceedings of Inter-Noise 88, pp. 1061-1064. ${ }^{8}$ B.-T. Wang, E. K. Dimitriadis, and C. R. Fuller, "Active control of panelradiated noise using multiple piezoelectric actuators," J. Acoust. Soc. Am. Suppl. 1 86, S84 (1989).

${ }^{9}$ B.-T. Wang, E. K. Dimitriadis, and C. R. Fuller, "Active control of structurally radiated noise using multiple piezoelectric actuators," in Proceedings of the AIAA/ASME/ASCE/AHS 31st Structures, Structural, Dynamics and Materials Conference, Long Beach, CA, 2-4 April 1990,
AIAA paper 90-1172.

${ }^{10} \mathrm{H}$. C. Lester and C. R. Fuller, "Active control of propeller induced noise fields inside a flexible cylinder," AIAA paper 86-1957 (1986).

"L. A. Roussos, "Noise transmission loss of a rectangular plate in an infinite baffle," NASA Technical Rep. 2398 (1985).

${ }^{12}$ M. C. Junger and D. Feit, Sound, Structures and their Interaction, 2nd ed. (MIT Press, Cambridge, MA, 1986), pp. 119-126.

${ }^{13}$ R. L. Clark, C. R. Fuller, and A. L. Wicks, "Characterization of multiple piezoelectric actuators for structural excitation," J. Acoust. Soc. Am. 90, 346-357 (1991).

${ }^{14}$ R. A. Burdisso and C. R. Fuller, "Theory of feed-forward controlled system eigenproperties," J. Sound Vib. (to be published).

${ }^{15}$ B.-T. Wang and C. R. Fuller, "Near-field pressure, intensity and wavenumber distributions for active structure control of plate radiation: Theoretical analysis," J. Sound Vib. (submitted).

${ }^{16} \mathrm{~L}$. Meirovitch and M. A. Norris, "Vibration control," in Proceedings of Inter-Noise 84, pp. 477-482. 